$11-1-2010$

\title{
Common Data Elements for Traumatic Brain Injury: Recommendations From the Biospecimens and Biomarkers Working Group
}

\author{
Geoffrey T. Manley \\ Ramon Diaz-Arrastia \\ Mary Brophy \\ Doortje Engel
}

Clay Goodman

See next page for additional authors

Follow this and additional works at: https://digitalcommons.cedarville.edu/

pharmaceutical_sciences_publications

Part of the Pharmacy and Pharmaceutical Sciences Commons

\begin{abstract}
This Article is brought to you for free and open access by DigitalCommons@Cedarville, a service of the Centennial Library. It has been accepted for inclusion in Pharmaceutical Sciences Faculty Publications by an authorized administrator of DigitalCommons@Cedarville. For more information, please contact digitalcommons@cedarville.edu.
\end{abstract}


Authors

Geoffrey T. Manley, Ramon Diaz-Arrastia, Mary Brophy, Doortje Engel, Clay Goodman, Katrina Gwinn, Timothy D. Veenstra, Geoffrey Ling, Andrew K. Ottens, Frank Tortella, and Ronald L. Hayes 


\title{
Common Data Elements for Traumatic Brain Injury: Recommendations From the Biospecimens and Biomarkers Working Group
}

\author{
Geoffrey T. Manley, PhD, Ramon Diaz-Arrastia, MD, PhD, Mary Brophy, MD, MPH, \\ Doortje Engel, MD, PhD, Clay Goodman, MD, Katrina Gwinn, MD, Timothy D. Veenstra, PhD, \\ Geoffrey Ling, MD, PhD, Andrew K. Ottens, PhD, Frank Tortella, PhD, Ronald L. Hayes, PhD
}

ABSTRACT. Manley GT, Diaz-Arrastia R, Brophy M, Engel D, Goodman C, Gwinn K, Veenstra TD, Ling G, Ottens AK, Tortella F, Hayes RL. Common data elements for traumatic brain injury: recommendations from the Biospecimens and Biomarkers Working Group. Arch Phys Med Rehabil 2010;91:1667-72.

Recent advances in genomics, proteomics, and biotechnology have provided unprecedented opportunities for translational research and personalized medicine. Human biospecimens and biofluids represent an important resource from which molecular data can be generated to detect and classify injury and to identify molecular mechanisms and therapeutic targets. To date, there has been considerable variability in biospecimen and biofluid collection, storage, and processing in traumatic brain injury (TBI) studies. To realize the full potential of this important resource, standardization and adoption of best practice guidelines are required to insure the quality and consistency of these specimens. The aim of the Biospecimens and Biomarkers Working Group was to provide recommendations for core data elements for TBI research and develop best practice guidelines to standardize the quality and accessibility of these specimens. Consensus recommendations were developed through interactions with focus groups and input from stakeholders participating in the interagency workshop on Standardization of Data Collection in TBI and Psychological Health held in Washington, DC, in March 2009. With the adoption of these standards and best practices, future investigators will be able to obtain data across multiple studies with reduced costs and effort and accelerate the progress of genomic, proteomic, and metabolomic research in TBI.

From the University of California San Francisco, San Francisco, CA (Manley); University of Texas Southwestern Medical School, Dallas, TX (Diaz-Arrastia); Department of Veterans Affairs Cooperative Studies Program, Boston, MA (Brophy); Heidelberg University Hospital, Heidelberg, Germany (Engel); Baylor College of Medicine, Houston, TX (Goodman, Gwinn); Science Applications International Corporation-Frederick Inc, National Cancer Institute at Frederick, Frederick, MD (Veenstra); Uniformed Services University, Bethesda, MD (Ling); Walter Reed Army Institute of Research, Silver Spring, MD (Tortella); Departments of Anatomy and Neurobiology, and Biochemistry, Virginia Commonwealth University, Richmond, VA (Ottens); Banyan Biomarkers Inc, Alachua, FL (Hayes).

A commercial party having a direct financial interest in the results of the research supporting this article has conferred or will confer a financial benefit on the author or one or more of the authors. R. L. Hayes is founder and president of Banyan Biomarkers Inc.

The views, opinions, or assertions contained herein are the private views of the authors and do not necessarily reflect those of the agencies or institutions with which they are affiliated, including the U.S. Department of Veterans Affairs, U.S. Department of Defense, Department of the Army, the U.S. Department of Health and Human Services, the National Institutes of Health, the National Institute of Mental Health, and the Uniformed Services University of the Health Sciences. This work is not an official document, guidance, or policy of the U.S. Government, nor should any official endorsement be inferred.

Correspondence to Geoffrey T. Manley, MD, PhD, University of California, San Francisco, Department of Neurosurgery, 1001 Potrero Ave, Building 1, Room 101, San Francisco, CA 94110, e-mail: manleyg@neurosurg.ucsf.edu. Reprints are not available from the author.

0003-9993/10/9111-00370\$36.00/0

doi:10.1016/j.apmr.2010.05.018

Key Words: Biological Markers; Brain Injuries; Rehabilitation. (C) 2010 by the American Congress of Rehabilitation Medicine

B ECAUSE GENOMIC AND PROTEOMIC research in TBI is still very much an emerging field, our goal was not to recommend assays of specific protein biomarkers or polymorphisms as core data elements. The aim of the working group was to provide recommendations for core biospecimen and biomarker data elements for TBI research and develop best practice guidelines to standardize the quality and accessibility of these specimens. We were also charged with establishing consensus for the core specimen data elements that should be collected for every TBI study and to identify supplemental and emerging data elements for more advanced and extended studies.

\section{APPROACH}

Multiple group conference calls were held as well as a number of smaller group calls and e-mail interactions. There was universal agreement on the need for standardization and development of best practices for collecting, processing, and storing biospecimens and biofluids. Past, current, and future efforts in civilian and military TBI biomarker genomics and proteomics research were reviewed and discussed.

Two series of observations support the fundamental hypothesis that genetic factors significantly influence functional outcome after TBI. The first of these is the finding that inheritance of the APOE-e4 allele is associated with poor outcome after TBI. ${ }^{1,2}$ The effect size of inheritance of one $A P O E-e 4$ allele is modest, with the odds ratio of an unfavorable outcome ranging from 3.57 to 13.93 . While these and other studies have primarily focused on 6-month outcome, ${ }^{3}$ there is evidence that the $A P O E-e 4$ genotype may also be associated with long-term outcome and cognitive decline after TBI. ${ }^{4}$ Thus, this polymorphism may be relevant in the patient with chronic as well as acute TBI. Polymorphisms in the interleukin-1 system have also been associated with outcome after TBI, but these studies have not so far been replicated. ${ }^{5}$ It is likely that the small sample sizes used in studies to date are a factor in false-

\section{List of Abbreviations}

$\begin{array}{ll}\text { APOE-e4 } & \text { apolipoprotein E4 } \\ \text { CDE } & \text { common data element } \\ \text { COMT } & \text { catechol O-methyltransferase } \\ \text { DNA } & \text { deoxyribonucleic acid } \\ \text { EDTA } & \text { ethylenediaminetetraacetic acid } \\ \text { EVD } & \text { external ventricular drain } \\ \text { LP } & \text { lumbar puncture } \\ \text { PBMC } & \text { peripheral blood mononuclear cell } \\ \text { RBC } & \text { red blood cell } \\ \text { TBI } & \text { traumatic brain injury }\end{array}$


negative and even false-positive findings. ${ }^{6}$ Although APOE-e4 appears to be the most likely candidate for the first "Core" genomic biomarker for TBI, future studies with sufficient patient numbers and comprehensive phenotyping are needed. There are also recent examples of discovery-driven proteomic studies focused on finding effective biomarkers for TBI. ${ }^{7,8}$ Mass spectrometry has been the primary proteomic technology and has revolutionized the ways that biomarker discovery is being conducted in this field.

Genomic biomarkers may also be relevant to psychologic health issues associated with TBI. For example, the COMT gene plays a key role in the degradation of dopamine in the frontal cortex. The Val ${ }^{158}$ Met polymorphism in the COMT gene has been associated with neuropsychiatric phenotypes and cognition. ${ }^{9,10}$ The $\mathrm{Val}^{158}$ Met genotype has also been associated with executive functioning in patients with TBI. ${ }^{11}$ Recent studies have also identified an association between serotonin transporter genotype and posttraumatic stress disorder, ${ }^{12}$ suggesting that this and other polymorphisms may help us understand the complicated interrelationship between TBI and psychologic health.

It was the consensus of the group that while there is significant potential for large-scale genomics and proteomics in TBI, there is sufficient variability in TBI biospecimen collection, processing, and storage that must be addressed to advance the field. ${ }^{13,14}$ Prospective clinical trials are an ideal setting in which to collect DNA and biological fluids in sufficiently large sample sizes associated with carefully collected clinical data to allow successful genomic and proteomic studies.

A due diligence process was carried out to explore similar efforts in other fields. A number of groups have already developed best practice guidelines for biospecimen resources. ${ }^{15-18}$ These documents provide general principles that can be used to guide the design of studies in which biospecimens will be analyzed. There was general agreement that much of the groundwork for the TBI biospecimen working group had been provided by these prior efforts. It was acknowledged that the group should not reinvent the wheel, but it was also recognized that there are issues particular to TBI that should be considered and addressed. The group felt that a document that built on past efforts and incorporated issues relevant to TBI would be an important contribution to the literature and the research community. Based on the members' individual expertise, the participants divided into subgroups to address various aspects of biofluid and biomarker specimen collection, storage, and processing. Preliminary recommendations were developed through e-mail interactions with the focus groups and discussed in person with the working group members at the interagency workshop on Standardization of Data Collection in TBI and Psychological Health held in Washington, DC, in March 2009. The refined draft recommendations and best practices were presented to stakeholders and other working groups during the workshop for additional input. These comments and suggestions have also been incorporated in the current recommendations. The product of the Biospecimens and Biomarkers Working Group is, by its nature, different from the other TBI CDE Working Group articles in that the focus is best practices with step-by-step protocols to standardize sample collection, processing, and storage.

\section{RECOMMENDATIONS FOR COMMON DATA ELEMENTS FOR BIOSPECIMENS AND BIOMARKERS}

The following are the group's recommendations for core specimen data elements that should be collected for all studies. The collection of supplemental and emerging data elements recommended for more advanced and extended studies should be encouraged, particularly in studies that are directed at a molecular mechanism that the biomarker measures.

\section{Core data element recommendations}

a. Collection of DNA sample for genomic analysis

b. Collection of acute $(<24 \mathrm{~h})$ plasma sample for proteomic and metabolomic analyses

2. Supplemental data element recommendations

a. Collection of serial plasma and serum samples for proteomic analysis

b. Collection of cerebrospinal fluid samples for proteomic analysis

3. Emerging data element recommendations

a. Collection of cerebral microdialysis samples

b. Collection of PBMCs for gene and protein expression studies

The TBI CDE Working Group recommendations for DNA guidelines for genomic analyses are presented in appendix 1 . The timing of DNA collection is less critical, even in patients who have received a blood transfusion. Recommendations for plasma and serum guidelines for proteomic and metabolomic analyses are shown in appendix 2. The timing for acquiring these samples is more complicated. An acute plasma sample (defined as $<24 \mathrm{~h}$ ) was recommended by the Working Group as "Core" for all TBI studies to provide the opportunity for the identification of diagnostic and predictive biomarkers in large series of patients. However, the frequency and duration of serial sample collection is biomarker-dependent and cannot be standardized at this time. Information regarding cerebrospinal fluid guidelines and microdialysis guidelines can be found in appendices 3 and 4, respectively. Each of these best practice guidelines addresses the acquisition, processing, and storage of the samples in sufficient detail to promote standardization.

\section{FUTURE DIRECTIONS}

Although these recommendations and best practices are voluntary, their adoption will lead to a new standardization that will advance TBI research. To demonstrate the utility of these recommendations and best practices, we propose a pilot study to examine the feasibility of multicenter biomarker CDE collection across the broad spectrum of TBI. Implementation of biospecimen and biomarker best practices will also require outreach and education to inform and engage the TBI research community and solicit comments and feedback. The working group believes that the recommendations and best practices for TBI biospecimens and biomarkers will continue to evolve as the field advances and potential biomarkers will one day become core CDEs. Thus, there will be a need for ongoing support for the TBI CDE effort.

\section{APPENDIX 1: DNA GUIDELINES FOR GENOMIC ANALYSES}

\section{Acquisition of blood biospecimens}

A. Blood is collected through venipuncture by appropriately trained personnel.

B. For isolation of genomic DNA from whole blood, collection of 5 to $20 \mathrm{~mL}$ using EDTA-containing Vacutainer tubes ${ }^{\mathrm{a}}$ is suitable. Use of citrate or heparinized tubes is also suitable.

C. Invert the sample 8 to 10 times to ensure proper mixture of blood and anticoagulant.

D. For isolation of PBMCs for future preparation of lymphoblastoid cell lines or gene expression studies, col- 


\section{APPENDIX 1 (Cont'd): DNA GUIDELINES FOR GENOMIC ANALYSES}

lection of 5 to $10 \mathrm{~mL}$ whole blood in LeucoPREP cell separation tubes. ${ }^{a}$ EDTA-containing vacutainer tubes may be used if the processing laboratory can perform Ficoll-Paque $^{\mathrm{b}}$ density gradient separation.

\section{Local processing}

A. Whole blood (in EDTA or LeucoPREP tubes) should be maintained at room temperature until transfer to the laboratory for processing. Studies have shown that packed cell volume starts decreasing with EDTA addition as early as 1 hour postcollection, so it is important to process the specimen in a time-efficient manner.

B. Transport the original, unfrozen Vacutainer without breaking the seal to the designated local genomics laboratory (if available). The Vacutainer system best preserves the integrity of the blood sample if it is not broken.

C. Because the expense of overnight shipping is justifiable only when highly specialized procedures such as preparation of lymphoblastoid cells lines are planned, the most cost-effective approach when such procedures are not required is to aliquot and freeze whole blood locally for DNA isolation later.

i. Storage in aliquots of $1-\mathrm{mL}$ to $2-\mathrm{mL}$ freezer-safe containers (ie, Cryovials) is most convenient.

ii. Multiple aliquots should be prepared in case of mishaps during shipment or DNA isolation. This also minimizes the freeze-thaw cycles should there be a need for multiple analyses at interval times.

D. DNA isolation from whole blood. It is most costefficient to isolate DNA from multiple (20-100 samples) at a time. This is most efficiently done by having each of the clinical sites store samples locally until a suitable number have been collected (ie, 10-20 samples). These can then be shipped in a single overnight shipment to a central laboratory experienced in collection and banking of samples.

E. Preparation of lymphoblastoid cell lines. Overnight shipment of LeucoPREP tubes to a laboratory experienced in the viral transformation and preparation of lymphoblastoid lines.

III. Local storage

A. Appropriate and complete documentation surrounding biospecimen collection, processing, and storage are essential and will influence the quality of research data to be obtained.

B. Samples should be placed in nonfrost-free freezers at or below $-80^{\circ} \mathrm{C}$. Frost-free freezers go through freezethaw cycles that further damage the specimen.

C. Avoid any thawing of frozen samples. Thawing of frozen samples of whole blood results in release of deoxyribinucleases that destroy the DNA.

D. Centers at which samples are stored should institute a back-up plan for freezer failure (eg, alternate power source, dry ice, or liquid nitrogen). An appropriate alarm system to support freezers for longtime storage is essential.

E. Lymphoblastoid cell lines must be stored in liquid nitrogen. The Coriell Institute provides storage for samples collected in National Institutes of Healthsponsored studies (http://www.coriell.org).

F. An inventory system should be established for tracking provenance of samples, including the time of collec-

\section{APPENDIX 1 (Cont'd): DNA GUIDELINES FOR GENOMIC ANALYSES}

tion, processing, storage, and quality control procedures carried out on each sample.

\section{Shipping}

A. Deoxyribinucleases degradation begins after 2 to 3 days at room temperature, so fresh blood samples should be shipped to the processing site within hours if possible. If the genomics laboratory is not within reach of local transport, frozen blood samples may be shipped through a designated agency.

B. Dry ice or cold packs must accompany the frozen specimen during air shipment. When dry ice is used, the transport time should be minimized given that dry ice sublimates at a rate of 2 to $5 \mathrm{~kg}$ per 24 hours depending on the insulation of the shipment container.

C. Consult the local agency for proper shipping options and certified transport materials. The International Air Transportation Association (http://www.iata.org) and the U.S. Department of Transportation (http://www.dot.gov) have legal requirements governing the packaging, labeling, and shipping of biospecimens.

i. Category A infectious substances are capable of causing permanent disability or life-threatening or fatal disease in humans or animals when exposure occurs.

ii. Category B infectious substances (also "diagnostic specimens" or "clinical specimens") are infectious but do not meet the standard for category A inclusion.

iii. Exempt patient specimens have a minimal likelihood of containing pathogens.

D. Temperature loggers can be used to monitor temperature in shipments of samples to provide confirmation and assurance that samples have been maintained at appropriate temperatures.

V. Central storage

A. Appropriate and complete documentation surrounding biospecimen collection, processing, storage, and shipping from the individual sites is essential and will influence the quality of the multicenter research data to be obtained.

B. Bar code identification of samples with an automated date and time stamp is recommended.

C. A formal plan for sharing the central biospecimen resource is recommended.

D. The Central Storage Bank should maintain information of laboratories where the samples have been sent to avoid duplicative genotyping and inadvertent repetitive reporting of data from the same patient.

E. The Central Storage Bank should also maintain information regarding any stipulations regarding informed consent for the use of the samples. For example, in some studies, participants may provide permission for their samples to be used only for studies on TBI.

\section{APPENDIX 2: PLASMA AND SERUM GUIDELINES FOR PROTEOMIC ANALYSES}

\section{Acquisition of blood biospecimens}

A. For severely injured patients, blood is collected via vascular access catheters that have been placed as part 


\section{APPENDIX 2 (Cont'd): PLASMA AND SERUM} GUIDELINES FOR PROTEOMIC ANALYSES

of the patients' routine medical care. For other patients, trained personnel collect blood through venipuncture.

B. For most purposes, 5 to $10 \mathrm{~mL}$ whole blood will be collected using a Vacutainer system. It should be noted that the use of glass tubes can lead to low values for certain analytes. For the most generalizable purposes, polypropylene Vacutainer and subsequent storage tubes are recommended.

\section{Local processing (serum)}

A. Blood samples should be collected in vacutainers that contain no anticoagulant for the processing of serum.

B. Samples should be sat upright at room temperature for 30 minutes to allow for clotting. They then should be spun at $4000 \mathrm{rpm}$ at room temperature for 5 to $7 \mathrm{~min}$ utes. The cleared serum should be pipetted and stored in aliquots of 1 to $2 \mathrm{~mL}$.

C. Record the volume of each aliquot.

III. Local processing (plasma)

A. Blood samples should be collected in vacutainers that contain EDTA when preparing plasma. Previous research suggests that EDTA is the preferred anticoagulant because others may interfere with analyte detection. ${ }^{19}$

i. The distinction between K2EDTA and K3EDTA and their concentrations should be assessed. See Goossens et $\mathrm{al}^{20}$ for more details.

B. Transport the original, unfrozen blood sample to the designated local proteomics laboratory as soon as possible. Freezing has significant adverse effects on plasma and its proteomic elements.

C. If specimens must be stored before processing, the samples should sit on ice for 5 to 10 minutes. Samples should be spun at 4000rpm at room temperature for 5 to 7 minutes. Plasma should be pipetted and stored in aliquots of $500 \mu \mathrm{L}$ to $2 \mathrm{~mL}$.

D. Record the volume of each aliquot.

IV. Local documentation and storage

A. Appropriate and complete documentation surrounding biospecimen collection, processing, and storage are essential and will influence the quality of research data to be obtained.

B. Bar code identification of samples with an automated date and time stamp is recommended.

C. Samples should be placed in nonfrost-free freezers at or below $-80^{\circ} \mathrm{C}$. Frost-free freezers go through freezethaw cycles that further damage the specimen.

D. Centers at which samples are stored should institute a back-up plan for freezer failure (eg, dry ice or liquid nitrogen). An appropriate alarm system to support freezers for longtime storage is essential.

E. An inventory system should be established for tracking provenance of samples, including the time of collection, processing, storage, and quality control procedures carried out on each sample.

\section{Shipping}

A. Dry ice or cold packs must accompany the frozen specimen during air shipment. When dry ice is used, the transport time should be minimized given that dry

\section{APPENDIX 2 (Cont'd): PLASMA AND SERUM} GUIDELINES FOR PROTEOMIC ANALYSES

ice sublimates at a rate of 2 to $5 \mathrm{~kg}$ per 24 hours depending on the insulation of the shipment container.

B. Consult the local agency for proper shipping options and certified transport materials. The International Air Transportation Association (http://www.iata.org) and the U.S. Department of Transportation (http://www.dot.gov) have legal requirements governing the packaging, labeling, and shipping of biospecimens.

i. Category A infectious substances are capable of causing permanent disability or life-threatening or fatal disease in humans or animals when exposure occurs.

ii. Category B infectious substances (also "diagnostic specimens" or "clinical specimens") are infectious but do not meet the standard for Category A inclusion.

iii. Exempt patient specimens have a minimal likelihood of containing pathogens.

D. Temperature loggers can be used to monitor temperature in shipments of samples to provide confirmation and assurance that samples have been maintained at appropriate temperatures.

VI. Central storage (see appendix 1)

\section{APPENDIX 3: CSF GUIDELINE}

\section{Acquisition of CSF from an EVD}

A. Document whether continuous or intermittent (catheter opened only in response to intracranial hypertension) fluid drainage is administered. Drainage method has been shown to alter CSF protein concentration. ${ }^{21}$

B. Draw CSF directly from ventriculostomy catheter.

C. Target CSF collection within the first 24 hours of admission, recording time from TBI and time of day. Ideally, the first collection should be as close to the TBI as feasible (eg, $6 \mathrm{~h}$ post-TBI), at a minimum frequency of every 6 hours for sufficient biokinetic studies.

D. Collect $5 \mathrm{~mL}$ of CSF in 1-mL fractions and place in ice bath. The first 1 or 2 fractions are sent for clinical laboratory analysis for cell count and protein and glucose measurements. Blood contamination of CSF is a significant confounder. Protein concentrations are 400fold greater in plasma than CSF. ${ }^{22} \mathrm{CSF}$ is considered blood-contaminated if $\mathrm{RBC}$ counts are greater than 10 cells $/ \mu \mathrm{L}$ or if hemoglobin levels are greater than $30 \mathrm{pg} / \mathrm{mL} \mathrm{CSF}{ }^{23}$ Brain specific proteins are typically present at low concentrations in CSF, with $80 \%$ of normal CSF protein mass originating from plasma. ${ }^{24}$

E. Appropriate CSF control samples may be available from hydrocephalic patients who undergo ventriculoperitoneal shunt placement and had CSF collected intraoperatively, or patients with unruptured subarachnoid hemorrhage who had CSF drawn intraoperatively. ${ }^{25}$

\section{Acquisition of CSF from an LP}

A. In patients who are unlikely to receive a ventriculostomy, CSF can be accessed by a less invasive LP. A greater number of LP collected control samples are available; however, comparison with EVD CSF is discouraged given a 2.5 -fold lower protein concentration than in LP CSF. ${ }^{26}$ 
APPENDIX 3 (Cont'd): CSF GUIDELINE

B. Atraumatic spinal needle LP kits should be used to minimize risk of post-LP headache. Draw with a sterile polypropylene syringe or allow flow under gravity.

C. Target CSF collection within the first 24 hours after admission, recording time from TBI and time of day. Ideally, the first collection should be as close to the TBI as feasible (eg, $6 \mathrm{~h}$ post-TBI) at a minimum frequency of every 6 hours for sufficient biokinetic studies.

D. Collect 1-mL fractions and place in ice bath, with a maximum of $25 \mathrm{~mL}$ a time point. Send the first $2 \mathrm{~mL}$ for clinical laboratory analysis. It is important to match fractions when comparing across patients because protein concentration varies depending on the draw volume. ${ }^{27}$

E. Patient should rest in a recumbent position for 1 hour post-LP, receive liberal fluid intake, and avoid exertion for 24 to 48 hours to minimize risk of headache.

\section{Processing and storage of CSF}

A. Transport CSF on ice and process immediately after collection because significant cell lysis contamination will occur within 1 hour.

B. Collect CSF samples into low protein binding polypropylene tubes (eg, Eppendorf brand LoBind tubes ${ }^{\mathrm{c}}$ ). Avoid polystyrene and glass tubes, which will result in significant protein loss. ${ }^{28}$

C. Centrifuge CSF at $2000 g$ and $4^{\circ} \mathrm{C}$ for 10 minutes. Draw off supernatant and place in new, low binding tube, and document volume of fluid collected. Centrifugation will only remove RBCs, not serum-derived proteins. Samples found to contain greater than $10 \mathrm{RBCs} / \mu \mathrm{L}$ CSF may be inappropriate for proteomic analysis.

D. Control samples are available; however, comparison with EVD CSF is discouraged given a 2.5 -fold lower protein concentration than in LP CSF. ${ }^{26}$

E. Additives or preservatives may be combined with CSF depending on experimental objectives. Protease and phosphatase inhibitors are recommended.

F. Snap freeze samples in liquid nitrogen, and store at or below $-80^{\circ} \mathrm{C}$ to minimize proteolytic breakdown. ${ }^{29}$ Avoid repeated freeze and thawing cycles and storage at $-20^{\circ} \mathrm{C}$.

\section{APPENDIX 4: CEREBRAL MICRODIALYSIS GUIDELINES}

I. Probe placement

A. In TBI, placement in pericontusional at-risk tissue is recommended, and if the option for placement of a second probe is available, the second probe should be placed in normal tissue.

B. Probe placement directly into contusions is of no value.

C. In case of diffuse injury, probe placement should be in the right frontal lobe.

II. Probe characteristics

A. Concentric configuration commercially available probes are preferable to locally fabricated probes.

B. Low-molecular-weight cutoff probes have been in use for many years and are available worldwide. ${ }^{31}$

C. Higher-molecular-weight cutoff probes are available but have not passed regulatory clearance in all jurisdictions, including the United States.

D. Probe molecular weight cutoff, manufacturer, and model must be specified in all data reporting.

\section{APPENDIX 4 (Cont'd): CEREBRAL} MICRODIALYSIS GUIDELINES

\section{Microdialysate characteristics}

A. Either of the following microdialysate fluids is acceptable:

i. Artificial CSF

ii. Sterile medical-grade normal saline

B. The composition and source of the microdialysate must be specified in all data reporting.

IV. Sample acquisition

A. The microdialysate flow rate should be $0.3 \mu \mathrm{L} / \mathrm{min}$ so that it is theoretically possible to collect $18 \mu \mathrm{L}$ during 1 hour. This flow rate assures near $100 \%$ analyte recovery for common low-molecular-weight analytes.

B. Because of the extremely small sample volumes, the samples are sensitive to evaporation even though they are sealed in the microvials, and because microvials may contain different volumes, the effect of evaporation can vary between the samples. Characteristics of the collecting containers must be reported.

C. Samples should be analyzed and/or stored promptly.

V. Analytes

A. The minimal essential data set consists of glucose, pyruvate, and lactate, with use of the calculated lactate/pyruvate ratio.

B. Measurement of glycerol and glutamate is recommended.

C. Determination of other analytes including markers of neuroinflammation such as cytokines, nitrate and nitrite as surrogates for nitric oxide, structural proteins such as glial fibrillary acidic protein, neurofilament, and tau and stress reactants such as beta-amyloid are all experimental. Analysis of these and other potential biomarkers should be encouraged but not required.

VI. Analyte stability during storage

A. The essential low-molecular-weight analytes are stable at $-70^{\circ} \mathrm{C}$. The samples should be stored at this temperature in containers designed to minimize evaporative losses.

B. Analytes, particularly pyruvate, may not be stable at $-20^{\circ} \mathrm{C}$. Storage at this temperature is not acceptable with the exception of temporary holding for not more than 3 days.

C. Determination of other analytes including markers of neuroinflammation such as cytokines, nitrate and nitrite as surrogates for nitric oxide, structural proteins such as GFAP, neurofilament, and tau and stress reactants such as beta-amyloid are all experimental. Analysis of these and other potential biomarkers should be encouraged but not required.

VII. Handling of samples at the time of analysis

A. If the samples have been frozen, then they must be thawed prior to analysis. It is important to recognize physicochemical events that may occur in the process of thawing that might cause analytic errors. As the samples thaw, the liquid phase will initially contain a very high concentration of salt and analytes. As the thawing progresses, the solution will be diluted by the melting ice. During this process, there is a risk that the thawed samples are nonhomogeneous; therefore, it is recommended that the samples be thawed and then agitated or centrifuged to assure homogeneous distribution of analyte. 


\section{APPENDIX 4 (Cont'd): CEREBRAL MICRODIALYSIS GUIDELINES}

B. It may be desirable to thaw the samples rapidly in a heating cupboard at $+40^{\circ} \mathrm{C}$ for about 10 minutes. Longer times and/or higher temperatures should not be used because these may result in a risk for unacceptable evaporation.

C. Stored samples may be assayed using the batch analysis systems. However, if the low-volume samples sit for too long in the analyzer prior to analysis, unacceptable evaporation may occur. Calibration samples should be interspersed in the batch to detect a systematic elevation in analyte levels caused by evaporative loss.

VIII. Microdialysis data reporting

A. Analyte concentrations should be reported in International System (SI) units.

B. Ratios such as the lactate/pyruvate ratio are devoid of units.

\section{References}

1. Teasdale GM, Nicoll JAR, Murray G, Fiddes M. Association of apolipoprotein E polymorphism with outcome after head injury. Lancet 1997;350:1069-71.

2. Friedman G, Froom P, Sazbon L, et al. Apolipoprotein E-epsilon4 genotype predicts a poor outcome in survivors of traumatic brain injury. Neurology 1999;52: 244-8.

3. Zhou W, Xu D, Peng X, Zhang Q, Jai J, Crutcher KA. Metaanalysis of APOE4 allele and outcome after traumatic brain injury. J Neurotrauma 2008;25:279-90.

4. Isoniemi H, Tonovuo O, Portin R, Himanan L, Kairisto V. Outcome of traumatic brain injury after three decades-relationship to ApoE genotype. J Neurotrauma 2006;23:1600-9.

5. Hadjigeorgiou GM, Paterakis K, Dardiotis E, et al. IL-1RN and IL-1B gene polymorphisms and cerebral hemorrhagic events after traumatic brain injury. Neurology 2005;65:1077-82.

6. Diaz-Arrastia R, Baxter VK. Genetic factors in outcome after traumatic brain injury: what the Human Genome Project can teach us about brain trauma. J Head Trauma Rehabil 2006;21:361-74.

7. Prieto DA, Ye X, Veenstra TD. Proteomic analysis of traumatic brain injury: the search for biomarkers. Expert Rev Proteomics 2008;5:283-91.

8. Ottens AK, Kobeissy FH, Fuller BF, et al. Novel neuroproteomic approaches to studying traumatic brain injury. Prog Brain Res 2007;161:401-18.

9. Egan MF, Goldberg TE, Kolachana BS, Callicot JH, Mazzanti CM, Straub RE. Effect of COMT Val108/158Met genotype on frontal lobe function and risk for schizophrenia. Proc Natl Acad Sci U S A 2001;98:6917-22.

10. De Frias CM, Annerbrink K, Westberg L, Eriksson E, Adolfsson $\mathrm{R}$, Nilsson L. Cotechol O-methyltransferase $\mathrm{Val}^{158} \mathrm{Met}$ polymorphism is associated with cognitive performance in nondemented adults. J Cogn Neurosci 2005;17:1018-25.

11. Lipsky RH, Sparling MB, Ryan LM, et al. Association of COMT Val158Met genotype with executive functioning following traumatic brain injury. J Neuropsychiatry Clin Neurosci 2005;17:465-71.

12. Xie P, Kranzler HR, Poling J, et al. Interactive effect of stressful life events and the serotonin transporter 5-HTTLPR genotype on posttraumatic stress disorder diagnosis in 2 independent populations. Arch Gen Psychiatry 2009;66:1201-9.

13. Schrohl AS, Wurtz S, Kohn E, et al. Banking of biological fluids for studies of disease-associated protein biomarkers. Mol Cell Proteomics 2009;7:2061-6.

14. Teunissen CE, Petzold A, Bennett JL, et al. A consensus protocol for the standardization of cerebrospinal fluid collection and biobanking. Neurology 2009;73:1914-22.
15. National Cancer Institute best practices guidelines for biospecimen resources. Prepared by the National Cancer Institute, National Institute of Health, and U.S. Department of Health and Human Services. June 2007. NIH pub no. 07-6229A. Washington (DC): National Institutes of Health; 2007.

16. International Society for Biological and Environmental Repositories. Best practices for repositories I: collection, storage, and retrieval of human biological materials for research. Cell Preserv Technol 2005;3:5-48.

17. Organization for Economic Co-Operation and Development. OECD best practice guidelines for biological resource centres. OECD Publishing: 2007. Available at: http://www.oecd.org/ dataoecd/7/13/38777417.pdf. Accessed February 1, 2009.

18. Eiseman E, Bloom G, Brower J, et al. Case studies of existing human tissue repositories: "best practices" for a biospecimen resource for the genomic and proteomic era. Santa Monica: RAND Corp; 2003.

19. Vanderstichele H, Van Kerschaver E, Hesse C, et al. Standardization of measurement of beta-amyloid (1-42) in cerebrospinal fluid and plasma. Amyloid, 2007;7:245-58.

20. Goossens W, Van Duppen V, Verwilghen RL. K2- or K3-EDTA: the anticoagulant of choice in routine haematology? Clin Lab Haematol 1991;13:291-5.

21. Shore PM, Thomas NJ, Clark RSB, et al. Continuous versus intermittent cerebrospinal fluid drainage after severe traumatic brain injury in children: effect on biochemical markers. J Neurotrauma 2004;21:1113-22.

22. Maurer MH. Proteomics of brain extracellular fluid (ECF) and cerebrospinal fluid (CSF). Mass Spectrom Rev 2010;29:17-28.

23. Zhang J. Proteomics of human cerebrospinal fluid-the good, the bad, and the ugly. Proteomics Clin Appl 2007;1:805-19.

24. Bergquist J, Palmblad M, Wetterhall M, Hakanssaon P, Markides KE. Peptide mapping of proteins in human body fluids using electrospray ionization fourier transform ion cyclotron resonance mass spectrometry. Mass Spectrom Rev 2002;21:2-15.

25. Pineda JA, Lewis SB, Valadka AB, et al. Clinical significance of $\alpha$ II-spectrin breakdown products in cerebrospinal fluid after severe traumatic brain injury. J Neurotrauma 2007;24:254-66.

26. Huhmer AF, Biringer RG, Amato H, Fonteh AN, Harrington MG. Protein analysis in human cerebrospinal fluid: physiological aspects, current progress and future challenges. Dis Markers 2006;22:3-26.

27. Blennow K, Fredman P, Wallin A, Gottfries CG, Langstrom G, Svennerholm L. Protein analyses in cerebrospinal fluid. I. Influence of concentration gradients for proteins on cerebrospinal fluid/serum albumin ratio. Eur Neurol 1993;33:126-8.

28. Hesse C, Larsson H, Fredman P, et al. Measurement of apolipoprotein E (apoE) in cerebrospinal fluid. Neurochem Res 2000;25:511-7.

29. Wagner AK, Ren D, Conley YP, et al. Sex and genetic associations with cerebrospinal fluid dopamine and metabolite production after severe traumatic brain injury. J Neurosurg 2007;106:538-47.

30. Carrette O, Burkhard PR, Hughes S, Hochstrasser DF, Sanchez JC. Truncated cystatin $\mathrm{C}$ in cerebrospinal fluid: technical [corrected] artifact or biological process? Proteomics 2005;5:3060-5.

31. Shores KS, Knapp DR. Assessment approach for evaluating high abundance protein depletion methods for cerebrospinal fluid (CSF) proteomic analysis. J Proteome Res 2007;6:3739-51.

\section{Suppliers}

a. Becton DIckinson Labware, 1 Becton Dr, Franklin Lakes, NJ 07417.

b. GE Healthcare, 800 Centennial Ave, \#1 Piscataway, NJ 08854-3930.

c. Eppendorf AG, Barkhausenweg 1, 22339 Hamburg 22331, Hamburg, Germany. 